\title{
Communication: The structures of small cationic gas-phase platinum clusters
}

\author{
Dan J. Harding, ${ }^{1}$ Christian Kerpal, ${ }^{1}$ David M. Rayner, ${ }^{2}$ and André Fielicke ${ }^{1, a)}$ \\ ${ }^{1}$ Fritz-Haber-Institut der Max-Planck-Gesellschaft, Faradayweg 4-6, D-14195 Berlin, Germany \\ ${ }^{2}$ National Research Council of Canada, 100 Sussex Drive, Ottawa, Ontario K1A OR6, Canada
}

(Received 23 April 2012; accepted 22 May 2012; published online 4 June 2012)

\begin{abstract}
The structures of small platinum clusters $\mathrm{Pt}_{3-5}^{+}$are determined using far-infrared multiple photon dissociation spectroscopy of their argon complexes combined with density functional theory calculations. The clusters are found to have compact structures, and $\mathrm{Pt}_{4}^{+}$and $\mathrm{Pt}_{5}^{+}$already favor threedimensional geometries, in contrast to a number of earlier predictions. Challenges in applying density functional theory to 3rd row transition metal clusters are addressed. Preliminary calculations suggest that the effects of spin-orbit coupling do not change the favoured lowest-energy isomers. (C) 2012 American Institute of Physics. [http://dx.doi.org/10.1063/1.4726403]
\end{abstract}

Gas-phase clusters are promising model systems to help unravel the complex atomistic details of reactions occurring on real heterogeneous nano-catalysts, including the effects on the reactivity of particle size, shape or charge state. Determination of gas-phase cluster geometric and electronic structures is an important step towards this understanding, demonstrated by the recent interest in the structures of gold clusters. ${ }^{1-4}$ In contrast, despite platinum being much more widely used in catalysis than gold, there are no structural or spectroscopic characterizations of platinum clusters larger than the trimer. There have been significant theoretical efforts to investigate the structures of isolated platinum clusters, ${ }^{5-11}$ but so far these are inconclusive, predicting different lowenergy structures. Here, we present a combined experimental and computational study of small platinum clusters to determine the structures of $\mathrm{Pt}_{n}^{+}(n=3-5)$ and investigate what level of theory is necessary to treat these systems reliably.

The importance of platinum in catalysis has lead to gasphase platinum clusters being the subject of a number of experiments to probe their reactivity, ${ }^{12-19}$ which has been found to depend sensitively on cluster size and charge state. Little spectroscopic work has been undertaken on unsupported platinum clusters, though features consistent with two vibrational progressions, at $105 \pm 30$ and $225 \pm 30 \mathrm{~cm}^{-1}$, have been observed in the photoelectron spectrum of $\mathrm{Pt}_{3}^{-} .{ }^{20}$

Practical quantum chemical calculations of the structures and properties of metal clusters remain limited to density functional theory (DFT) for all but the smallest sizes. In many cases, DFT provides accurate results at reasonable computational costs but, because of the approximations introduced in many exchange-correlation functionals, it is necessary to test the results of DFT calculations against experimental results. Vibrational spectroscopy provides an ideal comparison, as it is sensitive to the overall shape and structure of the cluster and the strengths and force constants of the bonds. Additionally, vibrational spectra can be calculated without knowledge of the excited electronic states of the cluster.

a)Electronic mail: fielicke@ fhi-berlin.mpg.de.
For heavy elements, such as $\mathrm{Pt}$, relativistic effects can become important, influencing both the structure of their compounds and their chemistry. Consequently, these effects may need to be included to provide an accurate theoretical/computational description of the cluster. While the most important relativistic effects can be routinely treated by scalar relativistic effective core potentials (ECP) other effects, particularly spin-orbit coupling (SOC), are more challenging. Several groups have made predictions of Pt cluster structures using DFT (Refs. 5-11) and post-Hartree-Fock methods, ${ }^{21-23}$ with and without the inclusion of SOC. Błoński et al. ${ }^{10}$ have summarized the available theoretical data on small Pt clusters and there is no consensus as to the favoured structures, with a range of two- and three-dimensional isomers proposed, or of the importance of SOC on the favoured isomer. The relative energy differences reported for the different isomers are, in many cases, extremely small. Consequently, only small changes in the energies of the different isomers are needed to change their energetic ordering.

Vibrational spectra of the clusters were obtained in the range from 90 to $250 \mathrm{~cm}^{-1}$ by far-infrared multiple photon dissociation (FIR-MPD) spectroscopy using the messengeratom technique. The experimental details and data analysis have been described in detail previously. ${ }^{24,25}$ Briefly, argontagged platinum clusters were formed in a laser ablation cluster source using a mixture of argon $(0.25 \%)$ in helium. The thermalisation channel of the source was cooled to $148 \mathrm{~K}$. After expansion into vacuum and passage through a skimmer, the ion beam enters the extraction region of a reflectron timeof-flight mass spectrometer. Intense, tunable far-infrared radiation from the Free Electron Laser for Infrared eXperiments ${ }^{26}$ (FELIX) counterpropagates along the cluster beam, typically providing $20 \mathrm{~mJ}$ in a $10 \mu \mathrm{s}$ long macropulse with a bandwidth of $\sim 1 \%$ FWHM of the central wavelength. When the IR radiation is resonant with a vibrational transition in the cluster multiple photons can be absorbed, heating the cluster, leading to dissociation. Changes in the mass spectrum are monitored as a function of FELIX wavelength. The IR spectra are shown on a cross section scale (see Ref. 25 for details). 

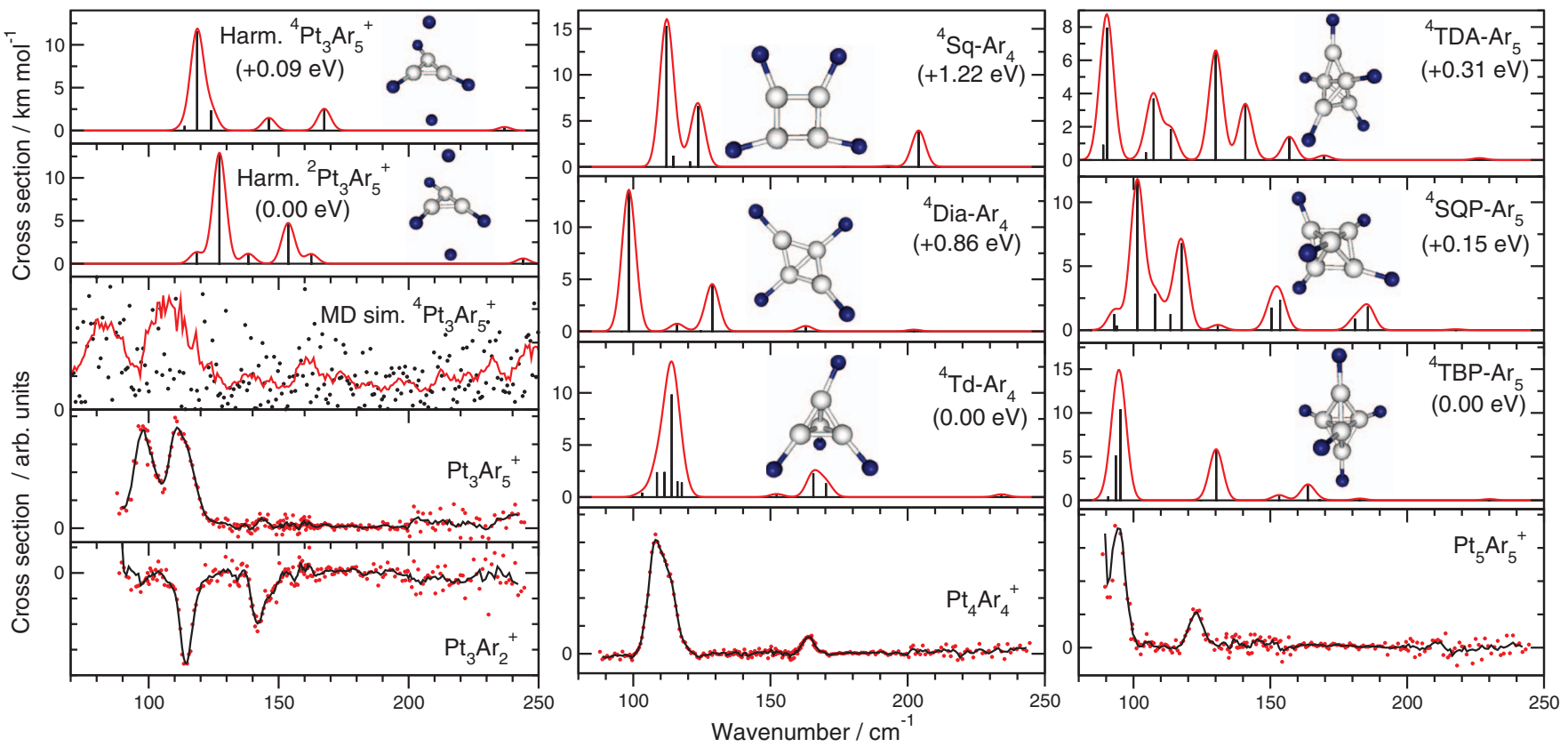

FIG. 1. Experimental and calculated spectra of $\mathrm{Pt}_{n} \mathrm{Ar}_{m}^{+}$. (a) $\mathrm{Pt}_{3} \mathrm{Ar}_{m}^{+}$; the experimental spectrum of $\mathrm{Pt}_{3} \mathrm{Ar}_{2}^{+}$shows growth due to fragmentation of clusters with more argon, below $\sim 100 \mathrm{~cm}^{-1}$ the heating of the clusters appears to be insufficient to boil off three $\mathrm{Ar}$ atoms from $\mathrm{Pt}_{3} \mathrm{Ar}_{5}^{+}$, (b) $\mathrm{Pt}_{4} \mathrm{Ar}_{4}^{+}$and (c) $\mathrm{Pt}_{5} \mathrm{Ar}_{5}^{+}$. All of the harmonic spectra were calculated at the TPSS/def2-TZVP level, the relative energies are shown for the argon complexes. The leading superscripts show the spin multiplicity of the species.

Argon sticks relatively strongly to these small platinum clusters and we can form and detect complexes even with the source at room temperature. The experimentally observed lifetime of the complexes of at least $100 \mu$ s sets an upper limit on the unimolecular dissociation rate of $10^{4} \mathrm{~s}^{-1}$. From the source temperature and the dissociation rate, using RiceRamsperger-Kassel-Marcus (RRKM) theory, we estimate the binding energy of the first Ar atom to be around $0.5 \mathrm{eV}$. A consequence of the strong binding is that the singly and doubly tagged complexes could not be dissociated. Instead, a higher concentration of argon was used and depletion of the complexes with four or five argon atoms was monitored. The tagging atoms necessary for the messenger-atom technique are usually assumed to cause only small changes to the vibrational spectra of the underlying molecule. When this is the case, the calculations needed for comparison can be performed without inclusion of the tagging atoms. Here, the relatively strongly bound Ar atoms may have a significant effect on the vibrational spectrum of the cluster complex and must therefore be explicitly included in the calculations.

The DFT calculations for bare and Ar-tagged Pt clusters were carried out using the TPSS exchange-correlation functional ${ }^{27}$ with the def2-TZVP ECP and basis sets ${ }^{28,29}$ using TURBOMOLE 6.0. ${ }^{30}$ To investigate the effects of SOC, we have performed calculations using NWChem $6.0,{ }^{31}$ with the TPSS functional ${ }^{27}$ and the CRENBL ECP, basis set and SO potential, ${ }^{32}$ which allows local optimization and frequency calculations with and without SOC. Currently, SOC calculations in NWChem cannot be performed with symmetry and IR intensities are not available, making the comparison with our experimental data more complicated. The results of the calculations for the bare clusters are shown in the supplementary material. ${ }^{33}$
A range of different geometries and spin-multiplicities ${ }^{34}$ were considered for each cluster size, the low-energy isomers were then decorated with argon and these structures reoptimised followed by frequency calculations. The frequencies are not scaled. As the clusters are charged, the most important interactions between the cluster and the Ar atoms are expected to be charge induced-dipole, rather than the non-local dispersion forces which are not treated by DFT.

Figure 1 shows the experimental FIR-MPD spectra and comparison with the calculated spectra for the Ar complexes of clusters containing three platinum atoms. $\mathrm{Pt}_{3} \mathrm{Ar}_{5}^{+}$has two intense bands at 98 and $111 \mathrm{~cm}^{-1}$ and a weak feature at $145 \mathrm{~cm}^{-1}$. Monitoring the $\mathrm{Pt}_{3} \mathrm{Ar}_{2}^{+}$channel, the lowest Arcoverage for which we see IR-induced intensity changes, we observe growth at 114 and $142 \mathrm{~cm}^{-1}$ due to fragmentation of $\mathrm{Pt}_{3} \mathrm{Ar}_{3-5}^{+}$. This growth, which appears as an apparent negative cross section, provides a composite spectrum for the clusters with higher Ar-coverage.

The bare $\mathrm{Pt}_{3}^{+}$cluster, despite its small size, presents a challenge to theory. The three levels of theory we have investigated, shown in Table I, all favour triangular structures but do not agree on the symmetries and spin multiplicities. We have also investigated linear isomers but found them to be higher in energy and, in many cases, saddle points. The calculated frequencies of the doublet and quartet triangular isomers are all fairly similar, with one mode of low or zero IR intensity above $220 \mathrm{~cm}^{-1}$, and two close (or degenerate) modes somewhere between 130 and $170 \mathrm{~cm}^{-1}$. As the band(s) observed in the experiment below $120 \mathrm{~cm}^{-1}$ are due to the Ar-tagging, the band at $142 \mathrm{~cm}^{-1}$ is the most useful for comparison with calculations of the bare clusters. Several of the spectra calculated for the triangular structures provide a reasonable match to the position of this band, the best is 
TABLE I. Calculated properties for triangular isomers of $\mathrm{Pt}_{3}^{+}$clusters at different levels of theory.

\begin{tabular}{cccc}
\hline \hline 2s +1 & $\begin{array}{c}\mathrm{E} \\
(\text { Hartree })\end{array}$ & $\begin{array}{c}\Delta \mathrm{E} \\
(\mathrm{eV})\end{array}$ & $\begin{array}{c}\text { Frequencies } \\
\left(\mathrm{cm}^{-1}\right)\end{array}$ \\
\hline \multicolumn{4}{c}{ TPSS/def2-TZVP } \\
2 & -357.683821 & 0.00 & $148,165,229$ \\
4 & -357.680889 & 0.08 & $145,146,219$ \\
6 & -357.625307 & 1.59 & $65,138,219$ \\
\multicolumn{4}{c}{ TPSS/CRENBL } \\
2 & -358.521039 & 0.12 & $121,121,250$ \\
4 & -358.525477 & 0.00 & $154,167,237$ \\
6 & -358.475738 & 1.35 & $128,146,234$ \\
\multicolumn{4}{c}{ TPSS/CRENBL with SOC } \\
2 & -358.915055 & 0.03 & $144,149,228$ \\
4 & -358.916141 & 0.00 & $134,143,233$ \\
6 & -358.916128 & 0.00 & $130,200,222$ \\
\hline \hline
\end{tabular}

provided by a doublet calculated with the inclusion of SOC, with modes at 144 and $149 \mathrm{~cm}^{-1}$. Unfortunately, similar changes in the calculated frequencies are caused by changes in ECP/basis set, spin multiplicity or geometry which, combined with the small energy differences and the complication of the Ar-tagging atoms, makes it difficult to distinguish between equilateral or lower symmetry triangles or doublet or quartet electronic states.

The spectra calculated with explicit inclusion of the Ar tagging atoms provide qualitatively good matches to the experiment, particularly to the growth seen in the $\mathrm{Pt}_{3} \mathrm{Ar}_{2}^{+}$channel. The frequencies around $150-170 \mathrm{~cm}^{-1}$ are due to metalmetal modes, while the intense low frequency bands are due to asymmetric Ar-cluster-Ar stretches. The band at $99 \mathrm{~cm}^{-1}$ is not reproduced by the harmonic spectra, even when taking the argon atoms into consideration. Given this problem, and the fact that some of the argon covered clusters have small imaginary frequencies $\left(\sim \mathrm{i} 10 \mathrm{~cm}^{-1}\right)$ where we have been unable to find true local minima due to the extremely flat nature of the potential energy surface, we have used molecular dynamics simulation with the FHI-AIMS program ${ }^{35}$ described in the supplementary material ${ }^{33}$ to investigate possible effects of anharmonicity or dynamics. For the ${ }^{4} \mathrm{Pt}_{3} \mathrm{Ar}_{5}^{+}$, the resulting spectrum, shown in Fig 1, is similar to the harmonic spectrum, with the exception of a second low-frequency band. Though this position of this feature does not match the experiment perfectly, it strongly suggests that the feature at $99 \mathrm{~cm}^{-1}$ is due to dynamic effects in the argon complex which cannot be described by the stationary harmonic spectra.

The experimental spectrum of $\mathrm{Pt}_{4} \mathrm{Ar}_{4}^{+}$, shown in Figure 1, has two features, at 110 and $164 \mathrm{~cm}^{-1}$. Both SOC and non-SOC calculations favour (roughly) tetrahedral (Td) geometries for the bare $\mathrm{Pt}_{4}^{+}$clusters. The calculated spectra of these low-energy structures provide a good match to the band positions of the experimental spectrum. If the argon atoms are included in the calculation, shown in Fig. 1, the intensities also match well. In this case, unlike $\mathrm{Pt}_{3} \mathrm{Ar}_{n}^{+}$, the bands at $110 \mathrm{~cm}^{-1}$ are not due solely to Pt-Ar modes but, at least in part, to metal-metal modes. While all three methods we have used find similar lowest-energy structures, the energy order and stability of other isomers are different. However, none of these isomers provide such a good match to the experimental spectrum. The most intense IR bands for the square (Sq) isomers are in the $190-200 \mathrm{~cm}^{-1}$ region, for the diamond isomers they are more variable, but they do not have intense features around $160 \mathrm{~cm}^{-1}$. In the case of the SOC calculations, the square isomers are all found to be transition states, while the diamond isomers collapse to $\mathrm{Td}$ structures during optimisation.

For bare $\mathrm{Pt}_{5}^{+}$clusters, we find quartet states of the square pyramid (SQP) and trigonal bipyramid (TBP) isomers to be basically isoenergetic at all three levels of calculation. The next best isomer, a tetrahedron with a bridge-bound adatom (TDA), is also relatively close in energy, from 0.05 to $0.2 \mathrm{eV}$ depending on the level of theory. We have also investigated several planar isomers but found these to be higher in energy $(0.5 \mathrm{eV})$. There are slightly larger differences in relative energies between the TPSS/CRENBL calculations with and without SOC than between the two sets of calculations without SOC (TPSS/CRENBL and TPSS/def2-TZVP) i.e., SOC appears to have a larger effect than the change of ECP and basis sets.

Including the Ar-atoms for the quartet states we find slightly larger energy differences than for the bare clusters, with ${ }^{4} \mathrm{TBP}^{-\mathrm{Ar}_{5}}$ now $0.15 \mathrm{eV}$ lower in energy than ${ }^{4} \mathrm{SQP}-$ $\mathrm{Ar}_{5}$. Comparison of the experimental and calculated spectra, shown in Fig. 1, finds a much better match for the ${ }^{4} \mathrm{TBP}-$ $\mathrm{Ar}_{5}$ than for the other isomers, matching the positions of the intense peaks at $<95$ and $123 \mathrm{~cm}^{-1}$. The small band at $165 \mathrm{~cm}^{-1}$ in the calculated spectrum is probably too weak for us to observe. The other calculated spectra do not provide good matches as they have too many features.

Given the very small energy differences predicted for at least three isomers of $\mathrm{Pt}_{5}^{+}$, it is surprising that we observe only one isomer, though the small number of features in the experimental spectrum also supports this interpretation. There are several possible reasons why this might be the case including (i) argon may stick preferentially to the TBP isomer, (ii) argon adsorption may lead to rearrangement of other isomers to the TBP, (iii) the DFT energy differences are too small and the TBP is significantly lower in energy than the other isomers.

The structures we have identified for $\mathrm{Pt}_{3-5}^{+}$are simple, close-packed geometries. They are similar to the structures we have identified for clusters of other transition metals. ${ }^{36,37}$ This is in agreement with structures predicted for the neutral clusters by post-Hartree-Fock ${ }^{21-23}$ and some $e^{5,7,9,11}$ of the DFT calculations. One possible (physical) source of difference is the charge state of the clusters, as our experiments and calculations are on cationic clusters, while the previous computational studies have investigated neutrals. For gold clusters, different charge states are known to favour different isomers ${ }^{1,3,4}$ and the transition from $2 \mathrm{D}$ to $3 \mathrm{D}$ isomers is observed at significantly smaller sizes for the cations than the anions. The adsorption of the Ar tagging atoms may also influence the observed isomer, though we have found no cases where the favored isomer is changed between the bare and $\mathrm{Ar}$ covered clusters.

We have tested the effects of some of the theoretical/computational choices which have to be made in order 
to do calculations on transition metals clusters, including the functional, ECP and basis set, or inclusion of SOC, and found they did not lead to significant changes in the lowest-energy structures for these very small Pt clusters. We have also measured spectra for larger clusters, where our preliminary calculations suggest SOC may become more important in determining the favoured geometry. The good agreement between the calculated spectra of the low-energy isomers and the experimental FIR-MPD spectra for all three sizes suggests that in this size range the TPSS functional recovers the correct lowest-energy structures and that SOC does not change the favoured isomer.

We gratefully acknowledge the support of the Stichting voor Fundamental Onderzoek der Materie (FOM) for providing FELIX beamtime and thank the FELIX staff, particularly Dr. A.F.G. van der Meer and Dr. B. Redlich, for their skillful assistance. We thank Gerard Meijer for his continued support. This work is funded by the Deutsche Forschungsgemeinschaft (DFG) through research Grant No. FI893/3-1. D.J.H. thanks the Alexander von Humboldt Foundation for a fellowship.

${ }^{1}$ S. Gilb, P. Weis, F. Furche, R. Ahlrichs, and M. M. Kappes, J. Chem. Phys. 116, 4094 (2002).

${ }^{2}$ J. Li, X. Li, H.-J. Zhai, and L.-S. Wang, Science 299, 864 (2003).

${ }^{3}$ M. P. Johansson, A. Lechtken, D. Schooss, M. M. Kappes, and F. Furche, Phys. Rev. A 77, 053202 (2008).

${ }^{4}$ P. Gruene, D. M. Rayner, B. Redlich, A. F. G. van der Meer, J. T. Lyon, G. Meijer, and A. Fielicke, Science 321, 674 (2008).

${ }^{5}$ T. Futschek, J. Hafner, and M. Marsman, J. Phys.: Condens. Matter 18, 9703 (2006)

${ }^{6}$ M. N. Huda, M. K. Niranjan, B. R. Sahu, and L. Kleinman, Phys. Rev. A 73, 053201 (2006).

${ }^{7}$ A. Sebetci, Chem. Phys. 331, 9 (2006).

${ }^{8}$ V. Kumar and Y. Kawazoe, Phys. Rev. B 77, 205418 (2008).

${ }^{9}$ A. Sebetci, Phys. Chem. Chem. Phys. 11, 921 (2009).

${ }^{10}$ P. Błoński, S. Dennler, and J. Hafner, J. Chem. Phys. 134, 034107 (2011).

${ }^{11}$ C. L. Herida, V. Ferraresi-Currotto, and M. B. López, Comput. Mater. Sci. 53, 18 (2012).

${ }^{12}$ D. J. Trevor, D. M. Cox, and A. Kaldor, J. Am. Chem. Soc. 112, 3742 (1990).

${ }^{13}$ U. Achatz, C. Berg, S. Joos, B. S. Fox, M. K. Beyer, G. NiednerSchatteburg, and V. E. Bondybey, Chem. Phys. Lett. 320, 53 (2000).
${ }^{14}$ T. Hanmura, M. Ichihashi, and T. Kondow, J. Phys. Chem. A 106, 11465 (2002).

${ }^{15}$ K. Koszinowski, D. Schröder, and H. Schwarz, J. Phys. Chem. A 107, 4999 (2003).

${ }^{16}$ I. Balteanu, O. P. Balaj, M. K. Beyer, and V. E. Bondybey, Phys. Chem. Chem. Phys. 6, 2910 (2004).

${ }^{17}$ G. Kummerlöwe, I. Balteanu, Z. Sun, O. P. Balaj, V. E. Bondybey, and M. K. Beyer, Int. J. Mass Spectrom. 254, 183 (2006).

${ }^{18}$ C. Adlhart and E. Uggerud, Chem. Commun. 2006, 2581.

${ }^{19}$ C. Adlhart and E. Uggerud, Chem.-Eur. J. 13, 6883 (2007)

${ }^{20}$ K. M. Ervin, J. Ho, and W. C. Lineberger, J. Chem. Phys. 89, 4514 (1988).

${ }^{21}$ D. Dai and K. Balasubramanian, J. Chem. Phys. 103, 648 (1995).

${ }^{22}$ D. Majumdar, D. Dai, and K. Balasubramanian, J. Chem. Phys. 113, 7919 (2000).

${ }^{23}$ D. Majumdar, D. Dai, and K. Balasubramanian, J. Chem. Phys. 113, 7928 (2000).

${ }^{24}$ A. Fielicke, A. Kirilyuk, C. Ratsch, J. Behler, M. Scheffler, G. von Helden, and G. Meijer, Phys. Rev. Lett. 93, 023401 (2004).

${ }^{25}$ A. Fielicke, G. von Helden, and G. Meijer, Eur. Phys. J. D 34, 83 (2005).

${ }^{26}$ D. Oepts, A. F. G. van der Meer, and P. W. van Amersfoort, Infrared Phys. Technol. 36, 297 (1995).

${ }^{27}$ J. Tao, J. P. Perdew, V. N. Staroverov, and G. E. Scuseria, Phys. Rev. Lett. 91, 146401 (2003).

${ }^{28}$ D. Andrae, U. Häußerman, M. Dolg, H. Stoll, and H. Preuß, Theor. Chim. Acta. 77, 123 (1990).

${ }^{29}$ F. Weigend and R. Ahlrichs, Phys. Chem. Chem. Phys. 7, 3297 (2005).

${ }^{30}$ R. Ahlrichs, M. Bär, M. Häser, H. Horn, and C. Kölmel, Chem. Phys. Lett. 162, 165 (1989).

${ }^{31}$ M. Valiev, E. Bylaska, N. Govind, K. Kowalski, T. Straatsma, H. V. Dam, D. Wang, J. Nieplocha, E. Apra, T. Windus, and W. de Jong, Comput. Phys. Commun. 181, 1477 (2010).

${ }^{32}$ R. B. Ross, J. M. Powers, T. Atashroo, W. C. Ermler, L. A. LaJohn, and P. A. Christiansen, J. Chem. Phys. 93, 6654 (1990).

${ }^{33}$ See supplementary material at http://dx.doi.org/10.1063/1.4726403 for details of the calculations for the bare clusters and the MD simulation.

${ }^{34}$ Spin contamination was a problem for doublet states in both sets of calculations performed without SOC, with $\left\langle S^{2}\right\rangle$ values suggesting a mix of doublet and quartet states. In some cases, we were able to find uncontaminated doublets by changing the orbital occupations, but these had significantly higher energies. In the SOC calculations, $\left\langle S^{2}\right\rangle$ need not be close to $S(S+1)$.

${ }^{35}$ V. Blum, R. Gehrke, F. Hanke, P. Havu, V. Havu, X. Ren, K. Reuter, and M. Scheffler, Comput. Phys. Commun. 180, 2175 (2009).

${ }^{36}$ A. Fielicke, C. Ratsch, G. von Helden, and G. Meijer, J. Chem. Phys. 127, 234306 (2007).

${ }^{37}$ A. Fielicke, P. Gruene, M. Haertelt, D. J. Harding, and G. Meijer, J. Phys. Chem. A 114, 9755 (2010). 